\title{
Thermodynamics of Rotating Black Branes in Gauss-Bonnet-Born-Infeld Gravity
}

\author{
M. H. Dehghani ${ }^{1,2 *}$ and S. H. Hendi ${ }^{1,3 \dagger}$ \\ ${ }^{1}$ Physics Department and Biruni Observatory, \\ College of Sciences, Shiraz University, Shiraz 71454, Iran \\ ${ }^{2}$ Research Institute for Astrophysics and Astronomy of Maragha (RIAAM), Maragha, Iran \\ 3 Physics Department, Yasouj University, Yasouj, Iran
}

\begin{abstract}
Considering both the Gauss-Bonnet and the Born-Infeld terms, which are on similar footing with regard to string corrections on the gravity side and electrodynamic side, we present a new class of rotating solutions in Gauss-Bonnet gravity with $k$ rotation parameters in the presence of a nonlinear electromagnetic field. These solutions, which are asymptotically anti-de Sitter in the presence of cosmological constant, may be interpreted as black brane solutions with inner and outer event horizons, an extreme black brane or naked singularity provided the metric parameters are chosen suitably. We calculate the finite action and conserved quantities of the solutions by using the counterterm method, and find that these quantities do not depend on the Gauss-Bonnet parameter. We also compute the temperature, the angular velocities, the electric charge and the electric potential. Then, we calculate the entropy of the black brane through the use of GibbsDuhem relation and show that it obeys the area law of entropy. We obtain a Smarr-type formula for the mass as a function of the entropy, the angular momenta and the charge, and show that the conserved and thermodynamic quantities satisfy the first law of thermodynamics. Finally, we perform a stability analysis in both the canonical and grand-canonical ensemble and show that the presence of a nonlinear electromagnetic field has no effect on the stability of the black branes, and they are stable in the whole phase space.
\end{abstract}

\footnotetext{
* email address: mhd@shirazu.ac.ir

$\dagger$ email address: hendi@mail.yu.ac.ir
} 


\section{INTRODUCTION}

In recent years a renewed interest in the Lovelock gravity and Born-Infeld electrodynamics has been appeared. This is due to the fact that both of them emerge in the low energy limit of string theory [1, 2, 3]. On the gravity side, string theories in their low-energy limit give rise to effective models of gravity in higher dimensions which involves higher curvature terms, while on the electrodynamics side the Born-Infeld action has been occurring with the development of superstring theory, where the dynamics of D-branes and some soliton solutions of supergravity is governed by the Born-Infeld action. While the Lovelock gravity was proposed to have field equations with at most second order derivatives of the metric [4], the nonlinear electrodynamics proposed, by Born and Infeld, with the aim of obtaining a

finite value for the self-energy of a point-like charge [5]. The Lovelock gravity reduces to Einstein gravity in four dimensions and also in the weak field limit, while the Lagrangian of the Born-Infeld (BI) electrodynamics reduces to the Maxwell Lagrangian in the weak field limit. There has been considerable works on both of these theories. In Lovelock gravity, there has been some attempts for understanding the role of the higher curvature terms from various points of view, especially with regard to black hole physics. For example, exact static spherically symmetric black hole solutions of the second order Lovelock (Gauss-Bonnet) gravity have been found in Ref. [6], and of the Gauss-Bonnet-Maxwell model in Ref. [7]. The thermodynamics of the uncharged static spherically black hole solutions has been considered in [8], of solutions with nontrivial topology in [9] and of charged solutions in [10, 11]. All of these known solutions in Gauss-Bonnet gravity are static. Not long ago one of us introduced two new classes of rotating solutions of second order Lovelock gravity and investigated their thermodynamics [12, 13], made the first attempt for finding exact static solutions in third order Lovelock gravity with the quartic terms [14], and presented the charged rotating black brane solutions of third order Lovelock gravity [15]. Recently, the Taub-NUT/bolt solutions of Gauss-Bonnet and Gauss-Bonnet-Maxwell gravity have been constructed [16, 17]. The first aim to relate the nonlinear electrodynamics and gravity has been done by Hoffmann [18]. He obtained a solution of the Einstein equations for a point-like Born-Infeld charge, which is devoid of the divergence of the metric at the origin that characterizes the ReissnerNordström solution. However, a conical singularity remained there, as it was later objected by Einstein and Rosen. The spherically symmetric solutions in Einstein-Born-Infeld (EBI) 
gravity with or without a cosmological constant have been considered by many authors [19, 20]. Recently, the rotating black brane solutions of Einstein-Born-Infeld gravity have been constructed, and their thermodynamics has been investigated.[21].

Considering the analogy between the Gauss-Bonnet and the Born-Infeld terms, which are on similar footing with regard to string corrections on the gravity side and electrodynamic side, respectively, it is plausible to include both these corrections simultaneously. Indeed, if one is to consider the Maxwell fields coupled to a gravitational action, which also includes string generated corrections at higher orders, then, it is naturally important to consider string generated corrections to the electromagnetic field action as well. Such corrections come from a coupling of abelian gauge fields to open bosonic or open superstrings. To be precise, it is known that, just like the Gauss-Bonnet terms, there are Born-Infeld terms which appear as higher order corrections to the Maxwell action [1]. The static black hole solutions of Gauss-Bonnet-Born-Infeld (GBBI) gravity have been constructed in Ref. [22]. In this paper, we attempt to construct the rotating black brane solutions of GBBI gravity and investigate their properties. We also perform a stability analysis and investigate the effects of nonlinear BI field on the stability of the solutions. The rest of the paper is organized as follows. In section II, we write down the first three term of Lovelock gravity (GaussBonnet) in $(n+1)$-dimensions with Born-Infeld type corrections of Maxwell equations. In section III, we consider a new class of rotating black brane and solve it for gravitation and electromagnetic field equations. We further discuss their thermodynamic properties in section IV. Section V has include of stability of black brane in canonical and grand-canonical ensemble. We finish our paper with concluding remarks in section IV.

\section{FIELD EQUATIONS IN GAUSS-BONNET-BORN-INFELD GRAVITY}

The most fundamental assumption in standard general relativity are the requirement of general covariance and that the field equations be second order. Based on these principles, the most general Lagrangian in arbitrary dimensions is the Lovelock Lagrangian. The gravitational action of Lovelock theory can be written as [4]

$$
I_{G}=\int d^{n+1} x \sqrt{-g} \sum_{i=0}^{[(n+1) / 2]} \alpha_{i} \mathcal{L}_{i}
$$


where $[z]$ denotes the integer part of $z, \alpha_{i}$ is an arbitrary constant and $\mathcal{L}_{i}$ is the Euler density of a $2 i$-dimensional manifold,

$$
\mathcal{L}_{i}=\frac{1}{2^{i}} \delta_{\rho_{1} \sigma_{1} \cdots \rho_{J} \sigma_{J}}^{\mu_{1} \nu_{1} \cdots \mu_{J} \nu_{J}} R_{\mu_{1} \nu_{1}}^{\rho_{1} \sigma_{1}} \cdots R_{\mu_{J} \nu_{J}}^{\rho_{J} \sigma_{J}}
$$

In Eq. (2),$\delta_{\rho_{1} \sigma_{1} \cdots \rho_{J} \sigma_{J}}^{\mu_{1} \nu_{1} \cdots \mu_{J} \nu_{J}}$ is the generalized totally anti-symmetric Kronecker delta and $R_{\mu \nu}{ }^{\rho \sigma}$ is the Riemann tensor. We note that in $(n+1)$-dimensions, all terms for which $d>[(n+1) / 2]$ are identically equal to zero, where $[x]$ denotes the integer part of $x$, and the term $d=$ $(n+1) / 2$ is a topological term. Consequently only terms for which $d<(n+1) / 2$ contribute to the field equations. Here we study Gauss-Bonnet gravity, that is first three terms of Lovelock gravity. In this case the action of GBBI gravity is

$$
\begin{aligned}
I_{G}= & -\frac{1}{16 \pi} \int_{\mathcal{M}} d^{n+1} x \sqrt{-g}\left\{R-2 \Lambda+\alpha\left(R_{\mu \nu \gamma \delta} R^{\mu \nu \gamma \delta}-4 R_{\mu \nu} R^{\mu \nu}+R^{2}\right)+L(F)\right\} \\
& -\frac{1}{8 \pi} \int_{\partial \mathcal{M}} d^{n} x \sqrt{-\gamma}\left\{\Theta+2 \alpha\left(J-2 \widehat{G}_{a b} \Theta^{a b}\right)\right\}
\end{aligned}
$$

where $\Lambda=-n(n-1) / 2 l^{2}$ is the cosmological constant, $\alpha$ is the Gauss-Bonnet coefficient with dimension (length) ${ }^{2}, R, R_{\mu \nu}$ and $R_{\mu \nu \gamma \delta}$ are the Ricci scalar and Ricci and Riemann tensors of the manifold $\mathcal{M}, L(F)$ is the Lagrangian of Born-Infeld

$$
L(F)=4 \beta^{2}\left(1-\sqrt{1+\frac{F^{2}}{2 \beta^{2}}}\right),
$$

In Eq. (41), $\beta$ is called the Born-Infeld parameter with dimension of mass, $F^{2}=F^{\mu \nu} F_{\mu \nu}$ where $F_{\mu \nu}=\partial_{\mu} A_{\nu}-\partial_{\nu} A_{\mu}$ is electromagnetic tensor field and $A_{\mu}$ is the vector potential. In the limit $\beta \rightarrow \infty, L(F)$ reduces to the standard Maxwell form $L(F)=-F^{2}$, while $L(F) \rightarrow 0$ as $\beta \rightarrow 0$. The second integral in Eq. (3) is a boundary term which is chosen such that the variational principle is well defined [23]. In this term, $\gamma_{a b}$ is induced metric on the boundary $\partial \mathcal{M}, \Theta$ is trace of extrinsic curvature $\Theta^{a b}$ of this boundary, $\widehat{G}^{a b}(\gamma)$ is Einstein tensor calculated on the boundary, and $J$ is trace of:

$$
J_{a b}=\frac{1}{3}\left(\Theta_{c d} \Theta^{c d} \Theta_{a b}+2 \Theta \Theta_{a c} \Theta_{b}^{c}-2 \Theta_{a c} \Theta^{c d} \Theta_{d b}-\Theta^{2} \Theta_{a b}\right)
$$

Varying the action with respect to the metric tensor $g_{\mu \nu}$ and electromagnetic field $A_{\mu}$ the equations of gravitation and electromagnetic fields are obtained as

$$
\begin{aligned}
& G_{\mu \nu}+\Lambda g_{\mu \nu}-\alpha\left\{4 R^{\rho \sigma} R_{\mu \rho \nu \sigma}-2 R_{\mu}^{\rho \sigma \lambda} R_{\nu \rho \sigma \lambda}-2 R R_{\mu \nu}+4 R_{\mu \lambda} R^{\lambda}{ }_{\nu}\right. \\
& \left.+\frac{1}{2} g_{\mu \nu}\left(R_{\kappa \lambda \rho \sigma} R^{\kappa \lambda \rho \sigma}-4 R_{\rho \sigma} R^{\rho \sigma}+R^{2}\right)\right\}=\frac{1}{2} g_{\mu \nu} L(F)+\frac{2 F_{\mu \lambda} F_{\mu}^{\lambda}}{\sqrt{1+\frac{F^{2}}{2 \beta^{2}}}}
\end{aligned}
$$




$$
\partial_{\mu}\left(\frac{\sqrt{-g} F^{\mu \nu}}{\sqrt{1+\frac{F^{2}}{2 \beta^{2}}}}\right)=0,
$$

where $G_{\mu \nu}$ is the Einstein tensor. Equation (6) does not contain the derivative of the curvatures, and therefore the derivatives of the metric higher than two do not appear. Thus, the Gauss-Bonnet gravity is a special case of higher derivative gravity.

In general the action $I_{G}$, is divergent when evaluated on the solutions, as is the Hamiltonian and other associated conserved quantities. A systematic method of dealing with this divergence in Einstein gravity is through the use of the counterterms method inspired by the anti-de Sitter conformal field theory (AdS/CFT) correspondence [24]. This conjecture, which relates the low energy limit of string theory in asymptotically anti de-Sitter spacetime and the quantum field theory living on the boundary of it, have attracted a great deal of attention in recent years. This equivalence between the two formulations means that, at least in principle, one can obtain complete information on one side of the duality by performing computation on the other side. A dictionary translating between different quantities in the bulk gravity theory and their counterparts on the boundary has emerged, including the partition functions of both theories. This conjecture is now a fundamental concept that furnishes a means for calculating the action and conserved quantities intrinsically without reliance on any reference spacetime [25]. It has also been applied to the case of black holes with constant negative or zero curvature horizons [26] and rotating higher genus black branes [27]. Although the AdS/CFT correspondence applies for the case of a specially infinite boundary, it was also employed for the computation of the conserved and thermodynamic quantities in the case of a finite boundary [28].

All of the work mention in the last paragraph was limited to Einstein gravity. Although the counterterms in Lovelock gravity should be a scalar constructed from Riemann tensor as in the case of Einstein gravity, they are not known for the case of Lovelock gravity till now. But, for the solutions with flat boundary, $\widehat{R}_{a b c d}(\gamma)=0$, there exists only one boundary counterterm

$$
I_{\mathrm{ct}}=-\frac{1}{8 \pi} \int_{\partial \mathcal{M}} d^{n} x \sqrt{-\gamma}\left(\frac{n-1}{l_{\mathrm{eff}}}\right),
$$

where $l_{\text {eff }}$ is a scale length factor that depends on $l$ and $\alpha$, that must reduce to $l$ as $\alpha$ goes to zero. One may note that this counterterm has exactly the same form as the counterterm in Einstein gravity for a spacetime with zero curvature boundary in which $l$ is replaced by 
$l_{\text {eff }}$ The total action can be written as a linear combination of the action of bulk, boundary (3) and the counterterm (8)

$$
I=I_{G}+I_{\mathrm{ct}}
$$

Having the total finite action, one can use the Brown-York definition of stress energy momentum tensor [29] to construct a divergence-free stress energy momentum tensor. For the case of manifolds with zero curvature boundary the finite stress energy momentum tensor is $[15]$

$$
T^{a b}=\frac{1}{8 \pi}\left\{\left(\Theta^{a b}-\Theta \gamma^{a b}\right)+2 \alpha\left(3 J^{a b}-J \gamma^{a b}\right)-\left(\frac{n-1}{l_{\mathrm{eff}}}\right) \gamma^{a b}\right\}
$$

One may note that when $\alpha$ goes to zero, the stress energy momentum tensor (10) reduces to that of Einstein gravity. To compute the conserved charges of the spacetime, we choose a spacelike surface $\mathcal{B}$ in $\partial \mathcal{M}$ with metric $\sigma_{i j}$, and write the boundary metric in ADM form:

$$
\gamma_{a b} d x^{a} d x^{a}=-N^{2} d t^{2}+\sigma_{i j}\left(d \varphi^{i}+V^{i} d t\right)\left(d \varphi^{j}+V^{j} d t\right)
$$

where the coordinates $\varphi^{i}$ are the angular variables parameterizing the hypersurface of constant $r$ around the origin, and $N$ and $V^{i}$ are the lapse and shift functions respectively. When there is a Killing vector field $\xi$ on the boundary, then the quasilocal conserved quantities associated with the stress energy momentum tensors of Eq. (10) can be written as

$$
\mathcal{Q}(\xi)=\int_{\mathcal{B}} d^{n-1} \varphi \sqrt{\sigma} T_{a b} n^{a} \xi^{b}
$$

where $\sigma$ is the determinant of the metric $\sigma_{i j}$, and $n^{a}$ is the timelike unit normal vector to the boundary $B$. For boundaries with timelike $(\xi=\partial / \partial t)$ and rotational $(\varsigma=\partial / \partial \varphi)$ Killing vector fields, one obtains the quasilocal mass and angular momentum

$$
\begin{aligned}
M & =\int_{\mathcal{B}} d^{n-1} \varphi \sqrt{\sigma} T_{a b} n^{a} \xi^{b}, \\
J & =\int_{\mathcal{B}} d^{n-1} \varphi \sqrt{\sigma} T_{a b} n^{a} \varsigma^{b},
\end{aligned}
$$

provided the surface $\mathcal{B}$ contains the orbits of $\varsigma$. These quantities are, respectively, the conserved mass and angular momentum of the system enclosed by the boundary $\mathcal{B}$. 


\section{THE $(n+1)$-DIMENSIONAL CHARGED ROTATING BLACK BRANES}

The metric of $(n+1)$-dimensional asymptotically AdS rotating spacetime with $k$ rotation parameters is [30, 31]

$$
\begin{gathered}
d s^{2}=-f(r)\left(\Xi d t-\sum_{i=1}^{k} a_{i} d \phi_{i}\right)^{2}+\frac{r^{2}}{l^{4}} \sum_{i=1}^{k}\left(a_{i} d t-\Xi l^{2} d \phi_{i}\right)^{2} \\
+\frac{d r^{2}}{f(r)}-\frac{r^{2}}{l^{2}} \sum_{i<j}^{k}\left(a_{i} d \phi_{j}-a_{j} d \phi_{i}\right)^{2}+r^{2} d X^{2}
\end{gathered}
$$

where $\Xi=\sqrt{1+\sum_{i}^{k} a_{i}^{2} / l^{2}}$ and $d X^{2}$ is the Euclidean metric on the $(n-1-k)$-dimensional submanifold. The rotation group in $(n+1)$ dimensions is $S O(n)$ and therefore $k \leq[n / 2]$. Using the gauge potential ansatz

$$
A_{\mu}=h(r)\left(\Xi \delta_{\mu}^{0}-\delta_{\mu}^{i} a_{i}\right)(\text { no sum on } i)
$$

and solving Eq. (7), we obtain

$$
h(r)=-\sqrt{\frac{n-1}{2 n-4}} \frac{q}{r^{n-2}}{ }_{2} F_{1}\left(\left[\frac{1}{2}, \frac{n-2}{2 n-2}\right],\left[\frac{3 n-4}{2 n-2}\right],-\eta\right),
$$

where $q$ is an integration constant which is related to the charge parameter, ${ }_{2} F_{1}([a, b],[c], z)$ is hypergeometric function and

$$
\eta=\frac{(n-1)(n-2) q^{2}}{2 \beta^{2} r^{2 n-2}}
$$

One may note that ${ }_{2} F_{1} \rightarrow 1$ as $\eta \rightarrow 0(\beta \rightarrow \infty)$ and $A_{\mu}$ of Eq. (16) reduces to the gauge potential of Maxwell field [12]. To find the function $f(r)$, one may use any components of Eq. (6). The simplest equation is the $r r$ component of these equations which can be written as

$$
\begin{aligned}
& (n-1)\left[r^{n-2}-2(n-2)(n-3) \alpha r^{n-4}\right] f^{\prime}+(n-1)(n-2)\left[r^{n-3}-(n-3)(n-4) \alpha r^{n-5} f\right] f \\
& +\left[2 \Lambda+2 \beta^{2}\left((1+\eta)^{1 / 2}-1\right)\right] r^{n-1}=0
\end{aligned}
$$

where the prime denotes a derivative with respect to $r$. The solutions of Eq. (18) can be written as

$$
f(r)=\frac{r^{2}}{2(n-2)(n-3) \alpha}(1-\sqrt{g(r)})
$$


where

$$
\begin{aligned}
g(r)= & \left(1-16 \frac{(n-3) \alpha \beta^{2} \eta}{n}{ }_{2} F_{1}\left(\left[\frac{1}{2}, \frac{n-2}{2 n-2}\right],\left[\frac{3 n-4}{2 n-2}\right],-\eta\right)\right) \\
& +4 \frac{(n-2)(n-3) \alpha}{n(n-1) r^{n}}\left(2 \Lambda r^{n}+n(n-1) m-4 \beta^{2} r^{n}+4 \beta^{2} r^{n} \sqrt{1+\eta}\right),
\end{aligned}
$$

Although the other components of the field Eq. (6) are more complicated, one can check that the metric (15) satisfies all the components of Eq. (6) provided $f(r)$ is given by (19, 201). Again, $f(r)$ reduces to the metric function of Ref. [12] as $\beta$ goes to infinity and reduces to that of Ref. [21], when $\alpha \longrightarrow 0$. Using the fact that ${ }_{2} F_{1}(a, b, c, z)$ has a convergent series expansion for $|z|<1$, one finds that $g(r)$ for large $\beta$ is

$$
\begin{aligned}
g(r)= & 1+\frac{8(n-2)(n-3) \alpha \Lambda}{n(n-1)}+\frac{4(n-2)(n-3) \alpha m}{r^{n}}-\frac{4(n-2)(n-3) \alpha q^{2}}{r^{2 n-2}} \\
& +\frac{(n-1)(n-2)^{3}(n-3) \alpha q^{4}}{2(3 n-4) \beta^{2} r^{4 n-4}}
\end{aligned}
$$

The last term in the right hand side of the Eq. (21) is the leading Born-Infeld correction to the Gauss-Bonnet-Maxwell black brane solutions.

\section{A. Properties of the solutions}

One can show that the above solution is asymptotically AdS with effective cosmological constant

$$
\Lambda_{\mathrm{eff}}=-\frac{n(n-1)}{4(n-2)(n-3) \alpha}\left(1-\sqrt{1-\frac{4(n-2)(n-3) \alpha}{l^{2}}}\right)
$$

As in the case of rotating black brane solutions of Einstein-Born-Infeld gravity, the above metric given by Eqs.(15, 19 and 20) has an essential singularity at $r=0$, and two types of Killing and event horizons. The Killing horizon is a null surface whose null generators are tangent to a Killing field. It is proved that a stationary black hole event horizon should be a Killing horizon in the four-dimensional Einstein gravity [32]. This proof can not obviously be generalized to higher order gravity, but the result is true for all the known static solutions. Although our solution is not static, the Killing vector,

$$
\chi=\partial_{t}+\sum_{i}^{k} \Omega_{i} \partial_{\phi_{i}}
$$

is the null generator of the event horizon, where $\Omega_{i}$ is the $i$ th component of angular velocity

of the outer horizon which may be obtained by analytic continuation of the metric. Setting 
$a_{i} \rightarrow i a_{i}$ yields the Euclidean section of (15), whose regularity at $r=r_{+}$requires that we should identify $\phi_{i} \sim \phi_{i}+\beta_{+} \Omega_{i}$. One obtains

$$
\Omega_{i}=\frac{a_{i}}{\Xi l^{2}}
$$

The Hawking temperature of the black brane is

$$
T=\frac{1}{2 \pi} \sqrt{-\frac{1}{2}\left(\nabla_{\mu} \chi_{\nu}\right)\left(\nabla^{\mu} \chi^{\nu}\right)}=\frac{r_{+}}{2(n-1) \pi \Xi}\left(2 \beta^{2}\left(1-\sqrt{1+\eta_{+}}\right)-\Lambda\right)
$$

Using the fact that the temperature of the extreme black brane is zero, it is easy to show that the condition for having an extreme black hole is that the mass parameter is equal to $m_{\text {ext }}$, where $m_{\text {ext }}$ is given as

$$
\begin{aligned}
m_{\mathrm{ext}}= & \frac{2(n-1) q_{\mathrm{ext}}^{n /(n-1)}}{n}\left[\frac{2\left(2 \beta^{2}-\Lambda\right)}{(n-1)^{2}}\left(\frac{(n-1)(n-2)}{2 \beta^{2} \eta_{\mathrm{ext}}}\right)^{n /(2 n-2)}+\right. \\
& \left.\left(\frac{(n-1)(n-2)}{2 \beta^{2} \eta_{\mathrm{ext}}}\right)^{-(n-2) /(2 n-2)}{ }_{2} F_{1}\left(\left[\frac{1}{2}, \frac{n-2}{2 n-2}\right],\left[\frac{3 n-4}{2 n-2}\right],-\eta_{\mathrm{ext}}\right)\right] ; \\
\eta_{\mathrm{ext}}= & \frac{\Lambda\left[\Lambda-4 \beta^{2}\right]}{4 \beta^{4}}
\end{aligned}
$$

The metric of Eqs.(15, 19 and 20) presents a black brane solution with inner and outer horizons, provided the mass parameter $m$ is greater than $m_{\text {ext }}$, an extreme black hole for $m=m_{\text {ext }}$ and a naked singularity otherwise. Note that the horizon radius of the extreme black brane is

$$
r_{+\mathrm{ext}}=\left(\frac{2(n-1)(n-2) \beta^{2} q_{\mathrm{ext}}^{2}}{\Lambda\left(\Lambda-4 \beta^{2}\right)}\right)^{1 /(2 n-2)}
$$

\section{THERMODYNAMICS OF BLACK BRANES}

In this section we, first, calculate the thermodynamic and conserved quantities of the black brane. Second, we obtain a Smarr-type formula for the mass as a function of the entropy, the angular momentum and the charge of the solution and check the first law of thermodynamics. Finally, we perform a stability analysis in both canonical and grandcanonical ensembles.

\section{A. Conserved and thermodynamic quantities}

Denoting the volume of the hypersurface at $r=$ constant and $t=$ constant by $V_{n-1}$, the charge per unit volume of the black brane, $Q$, can be found by calculating the flux of the 
electromagnetic field at infinity, yielding

$$
Q=\frac{(n-1)(n-2) \Xi}{8 \pi} q .
$$

The electric potential $\Phi$, measured at infinity with respect to the horizon, is defined by [33]

$$
\Phi=\left.A_{\mu} \chi^{\mu}\right|_{r \rightarrow \infty}-\left.A_{\mu} \chi^{\mu}\right|_{r=r_{+}},
$$

where $\chi$ is the null generator of the horizon given by Eq. (22). One finds

$$
\Phi=\sqrt{\frac{(n-1)}{2(n-2)}} \frac{q}{\Xi r_{+}^{n-2}}{ }_{2} F_{1}\left(\left[\frac{1}{2}, \frac{n-2}{2 n-2}\right],\left[\frac{3 n-4}{2 n-2}\right],-\eta\right) .
$$

Using Eqs. (13) and (14), the mass and angular momenta per unit volume $V_{n-1}$ of the solution are calculated as

$$
\begin{aligned}
M & =\frac{1}{16 \pi} m\left(n \Xi^{2}-1\right), \\
J_{i} & =\frac{1}{16 \pi} n \Xi m a_{i} .
\end{aligned}
$$

Black hole entropy typically satisfies the so-called area law, which states that the entropy of a black hole equals one-quarter of the area of its horizon [34]. This near universal law applies to almost all kinds of black holes and black strings in Einstein gravity [35]. However in higher derivative gravity the area law is not satisfied in general [36]. For asymptotically flat black hole solutions of [37]

$$
S=\frac{1}{4} \sum_{k=1}^{[(d-1) / 2]} k \alpha_{k} \int d^{n-1} x \sqrt{\tilde{g}} \tilde{\mathcal{L}}_{k-1}
$$

where the integration is done on the $(n-1)$-dimensional spacelike hypersurface of Killing horizon, $\tilde{g}_{\mu \nu}$ is the induced metric on it, $\tilde{g}$ is the determinant of $\tilde{g}_{\mu \nu}$ and $\tilde{\mathcal{L}}_{k}$ is the $k$ th order Lovelock Lagrangian of $\tilde{g}_{\mu \nu}$. The asymptotic behavior of the topological class of black holes we are considering is AdS, and therefore we calculate the entropy through the use of Gibbs-Duhem relation

$$
S=\frac{1}{T}\left(\mathcal{M}-\Gamma_{i} \mathcal{C}_{i}\right)-I
$$

where $I$ is the finite total action evaluated on the classical solution, and $\mathcal{C}_{i}$ and $\Gamma_{i}$ are the conserved charges and their associate chemical potentials respectively. Using eqs. (3) and (8), the finite action per unit volume $V_{n-1}$ can be calculated as

$$
I=-\frac{\Xi}{4 n}\left(r_{+}^{n-1}+\frac{2(n-1)^{2} l^{2} q_{2}^{2} F_{1}\left(\left[\frac{1}{2}, \frac{n-2}{2 n-2}\right],\left[\frac{3 n-4}{2 n-2}\right],-\eta_{+}\right)}{r_{+}^{n-1}\left[4 \beta^{2} l^{2}\left(1-\sqrt{1+\eta_{+}}\right)+n(n-1)\right]}\right)
$$


Now using Gibbs-Duhem relation (33) and eqs. (27) - (31) and (34) one obtains

$$
S=\frac{\Xi}{4} r_{+}^{(n-1)} .
$$

for the entropy per unit volume $V_{n-1}$. This shows that the entropy obeys the area law for our case where the horizon curvature is zero.

\section{B. Energy as a function of entropy, angular momenta and charge}

Calculating all the thermodynamic and conserved quantities of the black brane solutions, we now check the first law of thermodynamics for our solutions. We obtain the mass as a function of the extensive quantities $S, \mathbf{J}$, and $Q$. Using the expression for the entropy, the mass, the angular momenta, and the charge given in Eqs. (35), (27), (30), (31), and the fact that $f\left(r_{+}\right)=0$, one can obtain a Smarr-type formula as

$$
M(S, \mathbf{J}, Q)=\frac{(n Z-1) J}{n l \sqrt{Z(Z-1)}}
$$

where $J=|\mathbf{J}|=\sqrt{\sum_{i}^{k} J_{i}^{2}}$ and $Z=\Xi^{2}$ is the positive real root of the following equation:

$$
\begin{aligned}
& \left(n(n-1)+4 \beta^{2} l^{2}\right) S^{n /(n-1)}-4 \beta l^{2} S^{1 /(n-1)} \sqrt{\pi^{2} Q^{2}+\beta^{2} S^{2}}-\frac{4^{(n-2) /(n-1)}(n-1) \pi l J Z^{1 /(2 n-2)}}{\sqrt{(Z-1)}} \\
& +4 \frac{n-1}{n-2} \pi^{2} l^{2} Q^{2} S^{-(n-2) /(n-1)}{ }_{2} F_{1}\left(\left[\frac{1}{2}, \frac{n-2}{2 n-2}\right],\left[\frac{3 n-4}{2 n-2}\right],-\frac{\pi^{2} Q^{2}}{\beta^{2} S^{2}}\right)=0 .
\end{aligned}
$$

One may then regard the parameters $S, J_{i}$ 's, and $Q$ as a complete set of extensive parameters for the mass $M(S, \mathbf{J}, Q)$ and define the intensive parameters conjugate to them. These quantities are the temperature, the angular velocities, and the electric potential

$$
T=\left(\frac{\partial M}{\partial S}\right)_{J, Q}, \quad \Omega_{i}=\left(\frac{\partial M}{\partial J_{i}}\right)_{S, Q}, \quad \Phi=\left(\frac{\partial M}{\partial Q}\right)_{S, J} .
$$

It is a matter of straightforward calculation to show that the intensive quantities calculated by Eq. (38) coincide with Eqs. (23), (24), and (28). Thus, these quantities satisfy the first law of thermodynamics

$$
d M=T d S+\sum_{i=1}^{k} \Omega_{i} d J_{i}+\Phi d Q
$$




\section{Stability in the canonical and the grand-canonical Ensemble}

Finally, we investigate the stability of charged rotating black brane solutions of BornInfeld gravity. The stability of a thermodynamic system with respect to the small variations of the thermodynamic coordinates, is usually performed by analyzing the behavior of the entropy $S(M, Q, \mathbf{J})$ near equilibrium. The local stability in any ensemble requires that $S(M, Q, \mathbf{J})$ be a concave function of its extensive variables or that its Legendre transformation is a convex function of the intensive variables. The stability can also be studied by the behavior of the energy $M(S, Q, \mathbf{J})$ which should be a convex function of its extensive variable. Thus, the local stability can in principle be carried out by finding the determinant of the Hessian matrix of $M(S, Q, \mathbf{J})$ with respect to its extensive variables $X_{i}, \mathbf{H}_{X_{i} X_{j}}^{M}=\left[\partial^{2} M / \partial X_{i} \partial X_{j}\right]$ [33]. In our case the entropy $S$ is a function of the mass, the angular momenta, and the charge. The number of thermodynamic variables depends on the ensemble that is used. In the canonical ensemble, the charge and the angular momenta are fixed parameters, and therefore the positivity of the heat capacity $C_{\mathbf{J}, Q}=T_{+} /\left(\partial^{2} M / \partial S^{2}\right)_{\mathbf{J}, Q}$ is sufficient to ensure local stability. $\left(\partial^{2} M / \partial S^{2}\right)_{\mathbf{J}, Q}$ at constant charge and angular momenta is

$$
\begin{aligned}
\left(\frac{\partial^{2} M}{\partial S^{2}}\right)_{\mathrm{J}, Q}= & \Upsilon^{-1}\left\{\frac{(n-1) l^{2} m}{r_{+}^{n}}\left[(n-2) \Xi^{2}+1\right] \times\right. \\
& \left\{n(n-1) \sqrt{1+\eta_{+}}+4(n-2) l^{2} \beta^{2} \eta_{+}+4 l^{2} \beta^{2}\left(\sqrt{1+\eta_{+}}-1\right)\right\}+ \\
& n\left\{32 l^{4} \beta^{4}\left(\sqrt{1+\eta_{+}}-1\right)+n(n-1)\left(n(n-1) \sqrt{1+\eta_{+}}-8 l^{2} \beta^{2} \eta_{+}\right)+\right. \\
& \left.\left.8 n l^{2} \beta^{2}\left[2 l^{2} \beta^{2} \eta_{+}\left(\sqrt{1+\eta_{+}}-2\right)+n(n-1)\left(\sqrt{1+\eta_{+}}-1\right)\right]\right\}\left(\Xi^{2}-1\right)\right\} ; \\
\Upsilon= & (n-1)^{3} \pi m \Xi^{2} l^{4}\left[(n-2) \Xi^{2}+1\right]\left(1+\eta_{+}\right)^{1 / 2} r_{+}^{2}
\end{aligned}
$$

The heat capacity is positive for $m \geq m_{\text {ext }}$, where the temperature is positive. This fact can be seen easily for $\Xi=1$, where the second term of Eq. (39) is zero and the first term is positive. Also, one may see from Fig. [1 that the heat capacity increases as $\Xi$ increases, and therefore it is always positive. Thus, the black brane is stable in the canonical ensemble. In the grand-canonical ensemble, after some algebraic manipulation, we obtain

$$
H_{S \mathbf{J} Q}^{M}=\frac{\Pi}{\Psi}
$$




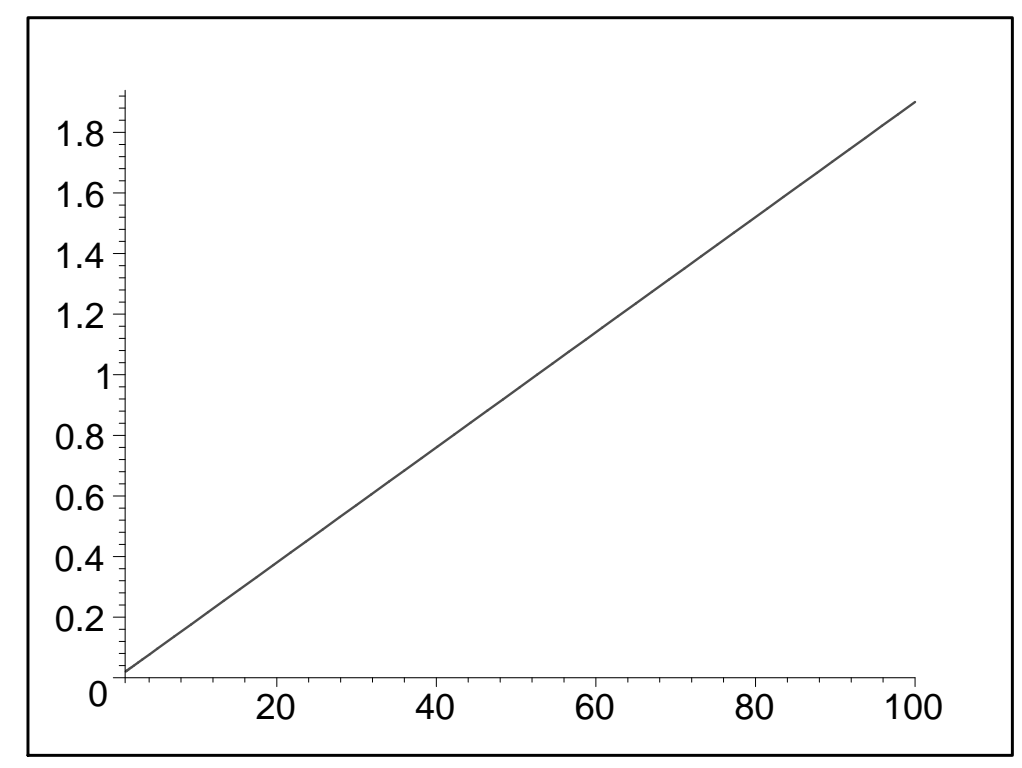

FIG. 1: $C_{\mathbf{J}, Q}$ versus $\Xi$ for $n=6, l=1, \beta=1, q=0.7$ and $r=0.8$.

where

$$
\begin{aligned}
\Pi= & \frac{64 \pi}{l^{2} \Xi^{6}\left[(n-2) \Xi^{2}+1\right]}\left\{\left(2 \beta^{2}\left(\left(1+\eta_{+}\right)^{1 / 2}-1\right)+(n-1)(n-2)^{2} q^{2} r_{+}^{-2 n+2}-\Lambda\left(1+\eta_{+}\right)^{1 / 2}\right)\right. \\
& \left.\times_{2} F_{1}\left(\left[\frac{1}{2}, \frac{n-2}{2 n-2}\right],\left[\frac{3 n-4}{2 n-2}\right],-\eta_{+}\right)+(n-2)\left(\left(2 \beta^{2}-\Lambda\right)-2 \beta^{2}\left(1+\eta_{+}\right)^{1 / 2}\right)\right\} \quad(41)
\end{aligned}
$$

and

$$
\begin{aligned}
\Psi= & (n-1)(n-2) r_{+}^{n-2}\left(1+\eta_{+}\right)^{1 / 2}\left\{(n-1)^{2} q_{2}^{2} F_{1}\left(\left[\frac{1}{2}, \frac{n-2}{2 n-2}\right],\left[\frac{3 n-4}{2 n-2}\right],-\eta_{+}\right)\right. \\
& \left.+r_{+}^{2 n-2}\left(\left(2 \beta^{2}-\Lambda\right)-2 \beta^{2}\left(1+\eta_{+}\right)^{1 / 2}\right)\right\},
\end{aligned}
$$

Since the value of ${ }_{2} F_{1}\left(\left[\frac{1}{2}, \frac{n-2}{2 n-2}\right],\left[\frac{3 n-4}{2 n-2}\right],-\eta_{+}\right)$is between 0 and 1 and $2 \beta^{2}\left(1+\eta_{+}\right)^{1 / 2} \leq$ $\left(2 \beta^{2}-\Lambda\right)$ for $q \leq q_{\text {ext }}$, it is easy to see that $H_{S \mathbf{J} Q}^{M}$ is positive for all the allowed values of

$$
q \leq q_{\mathrm{ext}}=\frac{r_{+}^{n-1}}{\beta} \sqrt{\frac{\Lambda\left(\Lambda-4 \beta^{2}\right)}{2(n-1)(n-2)}}
$$

Thus, the $(n+1)$-dimensional asymptotically AdS charged rotating black brane is locally stable in the grand-canonical ensemble. 


\section{CLOSING REMARKS}

In this paper, we found a new class of rotating solutions in Gauss-Bonnet-Born-Infeld gravity in the presence of the cosmological constant. These solutions which are asymptotically AdS and have flat horizon may be interpreted as black brane solutions with inner and outer event horizons provided the mass parameter $m$ is greater than an extremal value given by Eq.(25), an extreme black brane for $m=m_{\text {ext }}$ and a naked singularity otherwise. We found that these solutions reduce to the solutions of Eistein-Born-Infeld gravity as $\alpha \longrightarrow 0$, and reduce to those of Gauss-Bonnet-Maxwell gravity as $\beta \longrightarrow \infty$. The counterterm method inspired by the AdS/CFT correspondence has been widely applied to the case of Einstein gravity. Here, we applied this method to the solutions of Gauss-Bonnet-Born-Infeld gravity with flat boubdary at $r=$ constant and $t=$ constant, and calculated the finite action and conserved quantities of them. The physical properties of the brane such as the temperature, the angular velocity, the electric charge and the potential have been computed. We found that the conserved quantities of the black brane do not depend on the Gauss-Bonnet parameter $\alpha$. Then, we obtained the entropy of the black brane through the use of Gibbs-Duhem relation and found that it obeys the area law of entropy. We also obtained a Smarr-type formula for the mass as a function of the extensive parameters $S, \mathbf{J}$ and $Q$, calculated the intensive parameters, temperature, angular velocity, and electric potential, and showed that these quantities satisfy the first law of thermodynamics. We also studied the phase behavior of the $(n+1)$-dimensional rotating black branes in GBBI gravity and showed that there is no Hawking-Page phase transition in spite of the presence of a nonlinear electromagnetic field. Indeed, we calculated the heat capacity and the determinant of the Hessian matrix of the mass with respect to $S, \mathbf{J}$ and $Q$ of the black branes and found that they are positive for all the phase space, which means that the brane is stable for all the allowed values of the metric parameters discussed in Sec. IV] This phase behavior is commensurate with the fact that there is no Hawking-Page transition for a black object whose horizon is diffeomorphic to $\mathbb{R}^{p}$ and therefore the system is always in the high temperature phase [38]. Although, the presence of the nonlinear Born-Infeld field has no effects on the stability of the black brane, the presence of a scalar field makes the solution unstable [39]. 


\section{Acknowledgments}

This work has been supported by Research Institute for Astronomy and Astrophysics of Maragha, Iran

[1] E. S. Fradkin and A. A. Tseytlin, Phys. Lett. B 163, 123 (1985).

[2] E. Bergeshoeff, E. Sezgin, C. N. Pope and P. K. Townsend, Phys. Lett. B 188, 70 (1987).

[3] R. R. Metsaev, M. A. Rahmanov and A. A. Tseytlin, Phys. Lett. B 193, 207 (1987).

[4] D. Lovelock, J. Math. Phys. 12, 498 (1971); N. Deruelle and L. Farina-Busto, Phys. Rev. D 41, 3696 (1990); G. A. MenaMarugan, ibid. 46, 4320 (1992); ibid. 46, 4340 (1992).

[5] M. Born and L. Infeld, Proc. Roy. Soc. Lond. A 144, 425 (1934).

[6] D. G. Boulware and S. Deser, Phys. Rev. Lett. 55, 2656 (1985); J. T. Wheeler, Nucl. Phys. B 268, 737 (1986).

[7] D. L. Wiltshire, Phys. Lett. B 169, 36 (1986).

[8] R. C. Myers, Nucl. Phys. B 289, 701 (1987); R. C. Myers and J. Z. Simon, Phys. Rev. D 38, 2434 (1988).

[9] R. G. Cai, Phys. Rev. D 65, 084014 (2002); R. G. Cai and Q. Guo ibid. 69, 104025 (2004).

[10] M. Lu and M. B.Wise, Phys. Rev. D 47, 3095 (1993); M. Visser, ibid. 48, 583 (1993).

[11] M. Cvetic, S. Nojiri and S. D. Odintsov, Nucl. Phys. B 628, 295 (2002); S. Nojiri and S. D. Odintsov, Phys. Lett. B 521, 87 (2001).

[12] M. H. Dehghani, Phys. Rev. D 67, 064017 (2003).

[13] M. H. Dehghani, Phys. Rev. D 69, 064024 (2004); ibid. 70, 064019 (2004).

[14] M. H. Dehghani and M. Shamirzaie, Phys. Rev. D 72, 124015 (2005).

[15] M. H. Dehghani and R. B. Mann, Phys. Rev. D 73, 104003 (2006).

[16] M. H. Dehghani and R. B. Mann, Phys. Rev. D 72, 124006 (2005).

[17] M. H. Dehghani and S. H. Hendi, Phys. Rev. D 73, 084021 (2006).

[18] B. Hoffmann, Phys. Rev. 47, 877 (1935).

[19] M. Demianski, Found. Phys. 16, 187 (1986); H. d Oliveira, Class. Quant. Grav. 11, 1469 (1994); G. W. Gibbons and D. A. Rasheed, Nucl. Phys. B 454, 185 (1995).

[20] R.G. Cai, D.W. Pang and A. Wang, Phys.Rev. D 70, 124034 (2004); T. K. Dey, Phys. Lett. 
B 595, 484 (2004).

[21] M. H. Dehghani and H. Rastegar Sedehi, arXiv:hep-th/0610239.

[22] D. L. Wiltshire, Phys. Rev. D 38, 2445 (1988); M. Aiello, R. Ferraro and G. Giribet, Phys. Rev. D 70, 104014 (2004);

[23] R. C. Myers, Phys. Rev. D 36, 392 (1987); S. C. Davis, Phys. Rev. D 67, 024030 (2003).

[24] J. Maldacena, Adv. Theor. Math. Phys. 2, 231 (1998); E. Witten, ibid. 2, 253 (1998); O. Aharony, S. S. Gubser, J. Maldacena, H. Ooguri, and Y. Oz, Phys. Rep. 323, 183 (2000).

[25] M. Hennigson and K. Skenderis, J. High Energy Phys. 7, 023 (1998); V. Balasubramanian and P. Kraus, Commun. Math. Phys. 208, 413 (1999); S. Nojiri and S. D. Odintsov, Phys. Lett. B 444, 92 (1998); S. Nojiri, S. D. Odintsov, and S. Ogushi, Phys. Rev. D 62, 124002 (2000).

[26] M. H. Dehghani, Phys. Rev. D 66, 044006 (2002).

[27] M. H. Dehghani, Phys. Rev. D 65, 124002 (2002); R. G. Cai, ibid. 63, 124018 (2001); A. M. Ghezelbash D. Ida, R. B. Mann and T. Shiromizu, Phys. Lett. B 535, 315 (2002).

[28] M. H. Dehghani and R. B. Mann, Phys. Rev. D 64, 044003 (2001); M. H. Dehghani, ibid. 65, 104030 (2002); M. H. Dehghani and H. KhajehAzad, Can. J. Phys. 81, 1363 (2001).

[29] J. D. Brown and J. W. York, Phys. Rev. D 47, 1407 (1993).

[30] J. Lemos and V. Zanchin, Phys. Rev. D 54, 3840 (1996).

[31] A. M. Awad, Class. Quant. Grav. 20, 2827 (2003).

[32] S. W. Hawking, Commun. Math. Phys. 25, 152 (1972); S. W. Hawking and G. F. R. Ellis, The Large Scale Structure of Spacetime (Cambridge University Press, 1973).

[33] M. Cvetic and S. S. Gubser, J. High Energy Phys. 04, 024 (1999); M. M. Caldarelli, G. Cognola and D. Klemm, Class. Quantum Grav. 17, 399 (2000).

[34] J. D. Bekenstein, Phys. Rev. D 7, 2333 (1973); S. W. Hawking and C. J. Hunter, ibid. 59 044025 (1999); S. W. Hawking, C. J. Hunter and D. N. Page, ibid. 59, 044033 (1999); R. B. Mann, ibid. 60, 104047 (1999); ibid. 61, 084013 (2000).

[35] C. J. Hunter, Phys. Rev. D 59, 024009 (1999); R. B. Mann, ibid. 60, 104047 (1999); ibid. 61, $084013(2000)$.

[36] M. Lu and M. B. Wise, Phys. Rev. D 47, R3095,(1993); M. Visser, ibid. 48, 583 (1993).

[37] T. Jacobson and R. C. Myers, Phys. Rev. Lett. 70, 3684 (1993); R. M. Wald, Phys. Rev. D 48, 3427, (1993); M. Visser, ibid. 48, 5697 (1993); T. Jacobson, G. Kang and R. C. Myers, 
ibid. 49, 6587,(1994); V. Iyer and R. M. Wald, ibid. 50, 846 (1994).

[38] E. Witten, Adv. Theor. Math. Phys. 2, 505 (1998).

[39] A. Sheykhi, M. H. Dehghani, N. Riazi and J. Pakravan, Phys. Rev. D 74, 084016 (2006). 